\title{
Triglyceride Glucose Index Is Superior to the Homeostasis Model Assessment of Insulin Resistance for Predicting Nonalcoholic Fatty Liver Disease in Korean Adults
}

\author{
Sang Bae Lee ${ }^{1,2, *}$, Min Kyung Kim ${ }^{3, *}$, Shinae Kang ${ }^{1,2}$, Kahui Park ${ }^{1,2}$, Jung Hye Kim ${ }^{1,2}$, Su Jung Baik ${ }^{4}$, Ji Sun Nam ${ }^{1,2}$, \\ Chul Woo Ahn ${ }^{1,2}$, Jong Suk Park ${ }^{1,2}$ \\ ${ }^{1}$ Department of Internal Medicine, ${ }^{2}$ Severance Institute for Vascular and Metabolic Research, Yonsei University College of \\ Medicine; ${ }^{3}$ Department of Internal Medicine, Hallym University Kangdong Sacred Heart Hospital, Hallym University College \\ of Medicine; ${ }^{4}$ Healthcare Research Team of Health Promotion Center, Gangnam Severance Hospital, Yonsei University College \\ of Medicine, Seoul, Korea
}

Background: Recently, the triglyceride glucose (TyG) index has been considered a surrogate marker of insulin resistance which is a well-known pathogenic factor in nonalcoholic fatty liver disease (NAFLD). However, few studies have investigated the relationship between the TyG index and NAFLD. Thus, we investigated the relationship between the TyG index and NAFLD and the effectiveness of the TyG index compared with the homeostasis model assessment of insulin resistance (HOMA-IR) in identifying NAFLD in Korean adults.

Methods: Participants of 4,986 who underwent ultrasonography in a health promotion center were enrolled. The TyG index was calculated as $\ln$ [fasting triglycerides $(\mathrm{mg} / \mathrm{dL}) \times$ fasting glucose $(\mathrm{mg} / \mathrm{dL}) / 2$ ], and HOMA-IR was estimated. NAFLD was diagnosed by ultrasonography.

Results: Significant differences were observed in metabolic parameters among the quartiles of the TyG index. The prevalence of NAFLD significantly increased with increment in the TyG index. After adjusting for multiple risk factors, a logistic regression analysis was performed. When the highest and lowest quartiles of the TyG index and HOMA-IR were compared, the odds ratios for the prevalence of NAFLD were 2.94 and 1.93 (95\% confidence interval, 2.32 to 3.72 and 1.43 to 2.61 ; both $P$ for trend $<0.01$ ), respectively. According to the receiver operating characteristic analysis, the TyG index was superior to HOMA-IR in predicting NAFLD. Conclusion: The TyG index and prevalence of NAFLD were significantly related and the TyG index was superior to HOMA-IR in predicting NAFLD in Korean adults.

Keywords: Triglyceride glucose index; Insulin resistance; Non-alcoholic fatty liver disease

\section{INTRODUCTION}

Nonalcoholic fatty liver disease (NAFLD) is one of the most frequently occurring disorders of the liver, with the incidence gradually increasing worldwide [1,2]. NAFLD encompasses a range of liver diseases, including liver fibrosis, simple steatosis
Received: 12 February 2019, Revised: 26 March 2019, Accepted: 5 April 2019 Corresponding author: Jong Suk Park

Department of Internal Medicine, Gangnam Severance Hospital, Yonsei University College of Medicine, 211 Eonju-ro, Gangnam-gu, Seoul 06273, Korea

Tel: +82-2-2019-4377, Fax: +82-2-3463-3882, E-mail: pjs00@yuhs.ac *These authors contributed equally to this work.

\section{Copyright $(2019$ Korean Endocrine Society}

This is an Open Access article distributed under the terms of the Creative Commons Attribution Non-Commercial License (http://creativecommons.org/ licenses/by-nc/4.0/) which permits unrestricted non-commercial use, distribution, and reproduction in any medium, provided the original work is properly cited. 
and cirrhosis. Histopathologic changes associated with NAFLD may lead to liver failure, hepatocellular carcinoma and ultimately, hepatic mortality $[3,4]$. In recent years, the importance of NAFLD as a metabolic disorder has been realized, not only with regards to hepatic manifestation, but several studies have shown that NAFLD is also related to type 2 diabetes mellitus, abdominal obesity, dyslipidemia, hypertension, and cardiovascular disease [5-9]. Although the mechanisms associated with the onset of NAFLD remain poorly understood, insulin resistance (IR) has been found to be associated with the development of NAFLD [10-12]. The homeostasis model assessment of insulin resistance (HOMA-IR) is a widely used surrogate marker of IR and is one of several methods used for evaluating IR [13]. Numerous studies have found that an independent relationship exists between NAFLD and HOMA-IR [14-16]. In addition, other studies have proposed that HOMA-IR diagnostic criteria can be used for predicting NAFLD $[17,18]$.

Recently, the triglyceride glucose (TyG) index, which is calculated on the basis of triglycerides (TGs) and fasting glucose levels, has emerged as a reliable surrogate marker of IR. Moreover, the TyG index correlates with the HOMA-IR and hyperinsulinemic-euglycemic clamp test for recognizing IR [19-21]. Despite this, few studies have evaluated the TyG index in the context of NAFLD [22-24]. Therefore, in this study, we sought to elucidate the relationship between NAFLD and the TyG index and compare the effectiveness of the TyG index and HOMA-IR in identifying NAFLD in Korean adults.

\section{METHODS}

\section{Study participants}

A total of 5,989 Korean subjects (age $\geq 20$ years) who were participants in an in-depth health checkup program at the Gangnam Severance Hospital Health Promotion Center from January 2008 to February 2015, were included in the study. Subjects with elevated levels of TGs ( $\geq 400 \mathrm{mg} / \mathrm{dL})$, an acute inflammation, history of malignancy, renal or infectious disease, viral hepatitis (positive for hepatitis B surface antigen or anti-hepatitis $\mathrm{C}$ virus antibody), liver cirrhosis or malignancy observed via ultrasound were excluded from this study. Subjects with history of diabetes mellitus or newly diagnosed diabetes in this exam were also excluded. Furthermore, any subjects taking statins, TG-reducing therapies (e.g., fenofibrate or omega-3), thiazolidinediones, or injecting insulin were also excluded, as were males and females with a history of heavy alcohol consumption that exceeded 30 and $20 \mathrm{~g} /$ day, respectively. After the exclusion criteria were applied, 4,986 participants were included in the final analysis. All methods were performed in accordance with the ethical standards of the responsible committee on human experimentation and with the World Medical Association Declaration of Helsinki. Written informed consents were provided by all subjects before data collection. The study protocol was approved by the Institutional Review Board of Yonsei University College of Medicine (approval number: 2018-0077).

\section{Clinical characteristics of the study participants}

The height and weight of each participant was measured and the body mass index (BMI, $\mathrm{kg} / \mathrm{m}^{2}$ ) was calculated. The social and medical history of each participant was obtained by administering a self-questionnaire, which included questions regarding smoking, alcohol status, medications, and a history of other diseases. Experienced technicians measured the systolic blood pressure (SBP) and diastolic blood pressure (DBP) respectively, after a 5-minute of rest, with the patient's arm placed at the same level as the heart using an automated blood pressure monitor (HEM-7080IC, Omron Healthcare, Lake Forest, IL, USA). A diagnosis of diabetes mellitus was made on the basis of a prior history of diabetes or the American Diabetes Association's diagnostic standards. Subjects with SBP and/or DBP $\geq 140 / 90 \mathrm{~mm} \mathrm{Hg}$ or those presently using antihypertensive medication were defined as having hypertension. Subjects who had regularly smoked cigarettes over the past 6 months were considered to be current smokers.

\section{Biochemical parameters}

After an 8-hour fasting period, blood samples were collected from all subjects. The samples were immediately centrifuged and the serum was subsequently stored at $-70^{\circ} \mathrm{C}$ until further analysis was required. The levels of fasting plasma glucose (FPG), total cholesterol (TC), high density lipoprotein cholesterol (HDL-C), TG, aspartate aminotransferase (AST), and alanine aminotransferase (ALT) were assessed using enzymatic procedures, with an automated chemistry analyzer (Hitachi 7600-120, Hitachi, Tokyo, Japan). The level of low density lipoprotein cholesterol (LDL-C) was calculated using Friedewald formula. The TyG index was computed using the following formula: ln [fasting TGs $(\mathrm{mg} / \mathrm{dL}) \times$ fasting glucose $(\mathrm{mg} / \mathrm{dL}) / 2$ ] [19]. The levels of hepatitis B surface antigen and anti-hepatitis $\mathrm{C}$ virus antibodies were measured using a Roche E-170 device (Roche Diagnostics, Mannheim, Germany). The fasting serum insulin level was determined using a radioimmunoassay kit (Daiichi Radioisotope Labs, Tokyo, Japan). IR was approximat- 
ed using the HOMA-IR index which was calculated using following formula: [fasting insulin $(\mu \mathrm{U} / \mathrm{mL}) \times \mathrm{FPG}(\mathrm{mg} / \mathrm{dL}) / 405]$.

\section{Ultrasonographic analyses}

A diagnosis of fatty liver disease was made on the basis of the findings of an abdominal ultrasonography scan performed using a 3.5-MHz transducer (HDI 5000, Philips, Bothell, WA, USA). One of three experienced radiologists, who were blinded to the subjects' clinical information, performed the abdominal ultrasonographic examination. Any degree of fat accumulation in the liver was considered to be NAFLD in the present study. The subjects were classified into four groups on the basis of the existence and severity of NAFLD according to the level of hepatic tissue hyperechogenicity, discrepancy between the liver and right kidney, and visibility of the vascular structures [25].

\section{Statistical analysis}

Continuous variables with a normal distribution were presented as the mean \pm standard deviation. Continuous variables with skewed distributions were presented as the median with the interquartile range and were transformed to a log scale for analysis. Intergroup comparisons were performed using Student's $t$ test or one-way analysis of variance with post hoc analysis. Categorical variables with percentages were compared using chi-square test. After adjusting for any confounding variables, a multivariate logistic regression analysis was used for estimating the odds ratio (OR) and associated 95\% confidence interval (CI) for NAFLD on the basis of the TyG index and HOMA-IR. The area under the curve (AUC) of the receiver operating characteristic (ROC) curve was calculated for comparing the predictive power of the TyG index and HOMA-IR for the prevalence of NAFLD. $Z$ test was used for comparing the differences between AUCs. Statistical significance was considered for $P$ values less than 0.05 . SPSS for Windows version 23.0 (IBM Co., Armonk, NY, USA) and MedCalc (MedCalc software, Olstead, Belgium) were used for performing all statistical analyses in the present study.

\section{RESULTS}

A total of 4,986 subjects were included in the present study, 2,069 of whom were diagnosed with NAFLD using ultrasound data $(41.5 \%)$. The comparison of the baseline characteristics of the subjects enrolled in this study, with and without NAFLD, is presented in Table 1. The subjects with NAFLD were older;

Table 1. Clinical Characteristics of the Study Subjects Based on NAFLD Status

\begin{tabular}{lccc}
\hline Characteristic & All $(n=4,986)$ & No NAFLD $(n=2,917)$ & NAFLD $^{\mathrm{a}}(n=2,069)$ \\
\hline Age, $\mathrm{yr}$ & $52.63 \pm 9.26$ & $52.29 \pm 9.70$ & $53.11 \pm 8.58$ \\
Sex, male/female & $2,979 / 2,007$ & $1,422 / 1,495$ & $1,557 / 512$ \\
BMI, $\mathrm{kg} / \mathrm{m}^{2}$ & $23.82 \pm 3.17$ & $22.57 \pm 2.74$ & $25.59 \pm 2.87$ \\
SBP, $\mathrm{mm} \mathrm{Hg}$ & $124.97 \pm 16.55$ & $121.91 \pm 16.51$ & $129.28 \pm 15.61$ \\
DBP, $\mathrm{mm} \mathrm{Hg}$ & $77.91 \pm 10.20$ & $75.78 \pm 10.11$ & $80.90 \pm 9.55$ \\
FPG, $\mathrm{mg} / \mathrm{dL}$ & $93.93 \pm 10.70$ & $91.73 \pm 10.50$ & $97.04 \pm 10.19$ \\
TC, $\mathrm{mg} / \mathrm{dL}$ & $194.65 \pm 34.97$ & $191.88 \pm 34.00$ & $198.56 \pm 35.94$ \\
TG, $\mathrm{mg} / \mathrm{dL}$ & $96(69-139)$ & $83(61-114)$ & $120(87-168)$ \\
HDL-C, mg/dL & $50.20 \pm 12.75$ & $53.17 \pm 13.19$ & $46.02 \pm 10.79$ \\
LDL-C, $\mathrm{mg} / \mathrm{dL}$ & $120.21 \pm 31.21$ & $116.64 \pm 30.09$ & $125.24 \pm 32.07$ \\
AST, IU/L & $23.29 \pm 9.39$ & $22.03 \pm 8.44$ & $25.05 \pm 10.33$ \\
ALT, IU/L & $24.60 \pm 16.03$ & $20.82 \pm 12.59$ & $29.93 \pm 18.64$ \\
TyG index & $8.44 \pm 0.54$ & $8.28 \pm 0.51$ & $8.67 \pm 0.50$ \\
Insulin, $\mu \mathrm{IU} / \mathrm{mL}$ & $4.4(3.0-6.5)$ & $3.8(2.6-5.5)$ & $5.3(3.6-7.6)$ \\
HOMA-IR & $1.03(0.67-1.56)$ & $0.87(0.57-1.29)$ & $1.27(0.85-1.83)$ \\
Hypertension & $1,003(20.1)$ & $468(16.0)$ & $535(25.9)$ \\
\hline
\end{tabular}

Values are expressed as mean $\pm \mathrm{SD}$, median (interquartile range), or number (\%).

NAFLD, nonalcoholic fatty liver disease; BMI, body mass index; SBP, systolic blood pressure; DBP, diastolic blood pressure; FPG, fasting plasma glucose; TC, total cholesterol; TG, triglyceride; HDL-C, high density lipoprotein cholesterol; LDL-C, low density lipoprotein cholesterol; AST, aspartate aminotransferase; ALT, alanine aminotransferase; TyG, triglyceride glucose; HOMA-IR, homeostasis model assessment of assessment of insulin resistance. antergroup comparison using Student's $t$ test, all $P<0.01$. 
Table 2. Clinical Characteristics of the Study Participants Based on TyG Index

\begin{tabular}{|c|c|c|c|c|}
\hline Characteristic & Q1 $(n=1,249)$ & Q2 $(n=1,246)$ & Q3 $(n=1,245)$ & $\mathrm{Q} 4(n=1,246)^{\mathrm{a}}$ \\
\hline Age, yr & $51.13 \pm 9.98$ & $53.48 \pm 9.26^{\mathrm{b}}$ & $53.64 \pm 8.83^{\mathrm{c}}$ & $52.30 \pm 8.70^{\mathrm{d}, \mathrm{e}, \mathrm{f}}$ \\
\hline Sex, male/female & $475 / 774$ & $672 / 574$ & $842 / 403$ & $990 / 256$ \\
\hline BMI, $\mathrm{kg} / \mathrm{m}^{2}$ & $22.40 \pm 2.94$ & $23.49 \pm 3.15^{\mathrm{b}}$ & $24.20 \pm 2.98^{\mathrm{c}, \mathrm{g}}$ & $25.19 \pm 2.92^{\mathrm{d}, \mathrm{e}, \mathrm{f}}$ \\
\hline SBP, $\mathrm{mm} \mathrm{Hg}$ & $119.77 \pm 16.00$ & $123.87 \pm 16.69^{b}$ & $127.44 \pm 16.00^{\mathrm{c}, \mathrm{g}}$ & $128.80 \pm 16.01^{\mathrm{d}, \mathrm{e}}$ \\
\hline DBP, $\mathrm{mm} \mathrm{Hg}$ & $74.20 \pm 10.03$ & $77.06 \pm 10.02^{\mathrm{b}}$ & $79.58 \pm 9.72^{\mathrm{c}, \mathrm{g}}$ & $80.80 \pm 9.76^{\mathrm{d}, \mathrm{e}, \mathrm{f}}$ \\
\hline FPG, mg/dL & $86.81 \pm 9.31$ & $93.13 \pm 9.34^{b}$ & $96.56 \pm 9.90^{\mathrm{c}, g}$ & $99.26 \pm 9.98^{\mathrm{d}, \mathrm{ef}}$ \\
\hline $\mathrm{TC}, \mathrm{mg} / \mathrm{dL}$ & $184.80 \pm 33.25$ & $193.23 \pm 33.76^{\mathrm{b}}$ & $195.88 \pm 35.10^{\mathrm{c}}$ & $204.72 \pm 34.87^{\mathrm{d}, \mathrm{e}, \mathrm{f}}$ \\
\hline $\mathrm{TG}, \mathrm{mg} / \mathrm{dL}$ & $55(48-63)$ & $82(74-90)^{b}$ & $113(102-126)^{\mathrm{c}, \mathrm{g}}$ & $185(157-230)^{\mathrm{d}, \mathrm{e}, \mathrm{f}}$ \\
\hline HDL-C, mg/dL & $58.52 \pm 13.14$ & $52.43 \pm 12.05^{\mathrm{b}}$ & $47.28 \pm 10.62^{\mathrm{c}, \mathrm{g}}$ & $42.56 \pm 8.86^{\mathrm{de}, \mathrm{f}}$ \\
\hline LDL-C, mg/dL & $110.46 \pm 29.59$ & $121.46 \pm 30.20^{\mathrm{b}}$ & $124.39 \pm 31.31^{\mathrm{c}}$ & $124.57 \pm 31.59^{d}$ \\
\hline AST, IU/L & $21.94 \pm 8.40$ & $22.62 \pm 9.56$ & $23.30 \pm 9.49^{c}$ & $25.29 \pm 9.78^{\mathrm{d}, \mathrm{ef} \mathrm{f}}$ \\
\hline ALT, IU/L & $19.72 \pm 11.17$ & $22.80 \pm 16.25^{b}$ & $25.23 \pm 15.29^{\mathrm{c}, \mathrm{g}}$ & $30.67 \pm 18.49^{\text {de,ef }}$ \\
\hline TyG index & $7.76 \pm 0.21$ & $8.24 \pm 0.11^{b}$ & $8.60 \pm 0.11^{\mathrm{c}, \mathrm{g}}$ & $9.16 \pm 0.26^{\mathrm{de,f} f}$ \\
\hline Insulin, $\mu \mathrm{IU} / \mathrm{mL}$ & $3.0(2.2-4.6)$ & $4.1(2.9-5.9)^{b}$ & $4.7(3.5-6.7)^{\mathrm{c}, \mathrm{g}}$ & $5.7(3.9-8.0)^{\mathrm{d}, \mathrm{e}, \mathrm{f}}$ \\
\hline HOMA-IR & $0.65(0.45-0.99)$ & $0.93(0.66-1.34)^{b}$ & $1.11(0.79-1.62)^{\mathrm{c}, \mathrm{g}}$ & $1.40(0.29-1.93)^{\mathrm{d}, \mathrm{e}, \mathrm{f}}$ \\
\hline Hypertension & $164(13.1)$ & $232(18.6)$ & $287(23.1)$ & $320(25.7)$ \\
\hline
\end{tabular}

Values are expressed as mean $\pm \mathrm{SD}$, median (interquartile range), or number (\%).

TyG, triglyceride glucose; BMI, body mass index; SBP, systolic blood pressure; DBP, diastolic blood pressure; FPG, fasting plasma glucose; TC, total cholesterol; TG, triglyceride; HDL-C, high density lipoprotein cholesterol; LDL-C, low density lipoprotein cholesterol; AST, aspartate aminotransferase; ALT, alanine aminotransferase; HOMA-IR, homeostasis model assessment of insulin resistance.

${ }^{\mathrm{a}}$ Intergroup comparisons using one-way analysis of variance, all $P<0.01 ;{ }^{\mathrm{b}} P<0.05$ between $\mathrm{Q} 1$ and $\mathrm{Q} 2 ;{ }^{\mathrm{c}} P<0.05$ between $\mathrm{Q} 1$ and Q3; ${ }^{\mathrm{d}} P<0.05$ between Q1 and Q4; ${ }^{\mathrm{e}} P<0.05$ between Q2 and Q4; ${ }^{\mathrm{f}} P<0.05$ between $\mathrm{Q} 3$ and Q4; ${ }^{\mathrm{g}} P<0.05$ between Q2 and Q3.

more likely to be male; and had a higher BMI, blood pressure, insulin, HOMA-IR, LDL-C, FPG, TC, TG, AST, ALT, and TyG index but lower HDL-C than the subjects without NAFLD. In addition, the proportion of subjects with hypertension was significantly higher among those with NAFLD than those without.

The subjects were divided into four groups on the basis of their TyG indices (Q1: TyG index $\leq 8.04$; Q2: 8.05 $\leq$ TyG index $\leq 8.42$; Q3: 8.43 $\leq$ TyG index $\leq 8.81$; Q4: TyG index $\geq 8.82$ ). Table 2 outlines the demographic, clinical, and laboratory information for each group. The metabolic parameters were associated with significant differences between the groups. There was an increase in the number of males, BMI, SBP, DBP, AST, ALT, insulin, HOMA-IR, FPG levels, the prevalence of hypertension, as well as an increase in the levels of TC, TG, and LDL-C. However, the level of HDL-C was found to decrease with increasing TyG index.

The subjects were also classified into four groups according to their HOMA-IR (Q1: HOMA-IR $\leq 0.67$; Q2: $0.68 \leq$ HOMAIR $\leq 1.03$; Q3: $1.04 \leq$ HOMA-IR $\leq 1.56$; Q4: TyG index $\geq 1.57$ ) and results demonstrated that both the prevalence and severity of NAFLD increased with increasing TyG index and HOMA-IR quartiles (Fig. 1).

The association between the TyG index and NAFLD was investigated by dividing the TyG index into the following quartiles: the first quartile (Q1) was used as a reference (Table 3). When Q1 was set as a reference, the unadjusted multivariate logistic regression analysis revealed that in all subjects, the TyG indices for Q2, Q3, and Q4 were associated with higher OR for the presence of NAFLD. Moreover, these relationships remained significant $(P$ for trend $<0.01$ ) even after adjusting for confounding variables. The association between the HOMA-IR and the presence of NAFLD was further investigated by categorizing the HOMA-IR into quartiles, with the first quartile used as the reference (Table 3 ). Results showed that there was a significant relationship ( $P$ for trend $<0.01$ ) between the higher HOMA-IR quartile and the presence of NAFLD, even after adjusting for confounding variables. After adjusting for multiple risk factors, the OR $(95 \% \mathrm{CI})$ in the highest quartile for NAFLD using HOMA-IR was 1.93 (95\% CI, 1.43 to 2.61) compared with 2.94 (95\% CI, 2.32 to 3.72) for the TyG index. The AUC $(95 \% \mathrm{CI})$ of the ROC curve for the TyG index at 0.716 (95\% CI, 0.702 to 0.731 ) was significantly higher than that of the HOMA- 


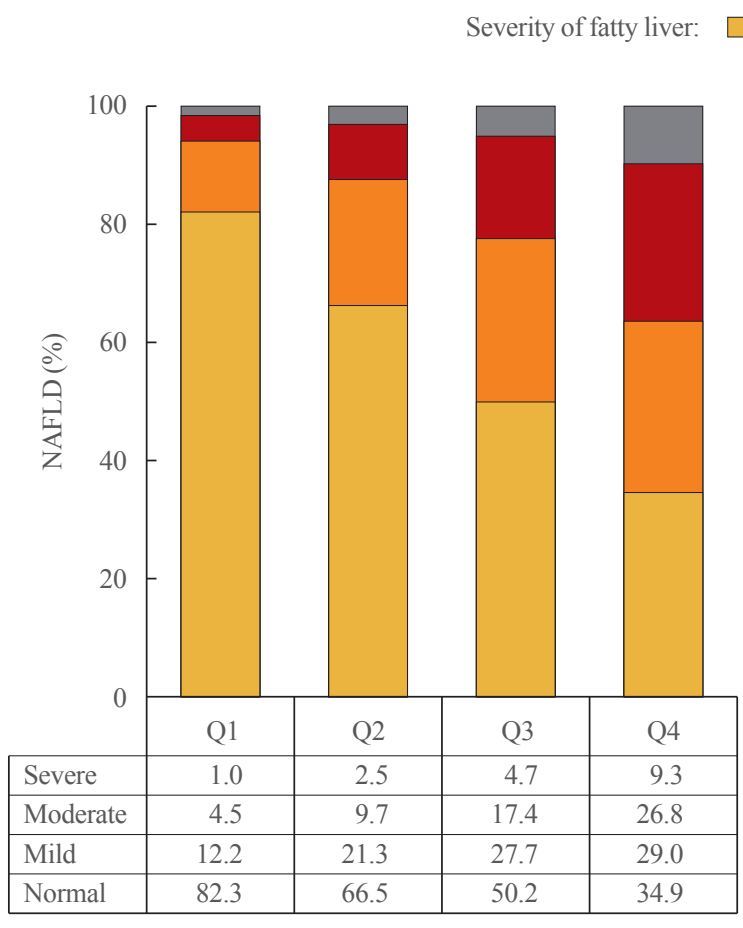

TyG index

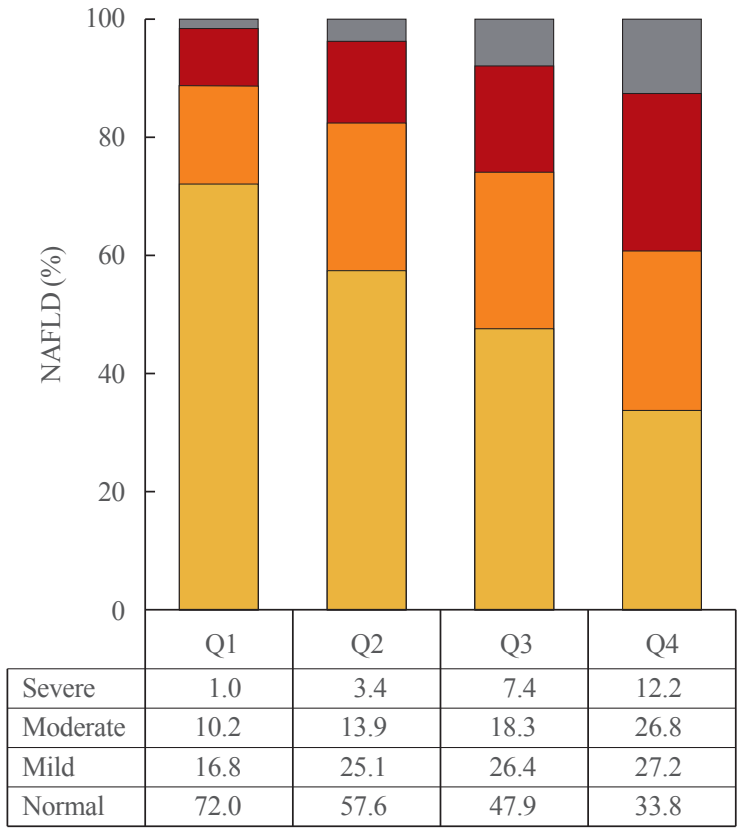

HOMA-IR

Fig. 1. The prevalence and severity of nonalcoholic fatty liver disease (NAFLD) based on the (A) triglyceride glucose (TyG) index and (B) homeostasis model assessment of insulin resistance (HOMA-IR). Ultrasonography was used for diagnosing the severity of fatty liver disease (all $P<0.01)$.

Table 3. Nonalcoholic Fatty Liver Disease OR and 95\% CI Based on the TyG Index and HOMA-IR Quartiles

\begin{tabular}{lcccc}
\hline & \multicolumn{3}{c}{ OR $(95 \%$ CI $)$} \\
\cline { 2 - 5 } & Q1 & Q2 & Q3 & Q4 $^{\mathrm{a}}$ \\
\hline TyG index & & & & \\
$\quad$ Unadjusted & 1.00 & $2.35(1.95-2.83)$ & $4.57(3.81-5.49)$ & $8.73(7.25-10.52)$ \\
$\quad$ Multivariable adjusted & 1.00 & $1.57(1.26-1.94)$ & $2.32(1.87-2.88)$ & $2.94(2.32-3.72)$ \\
HOMA-IR & & & & \\
Unadjusted & 1.00 & $1.88(1.46-2.42)$ & $2.93(2.28-3.76)$ & $5.15(3.98-6.66)$ \\
Multivariable adjusted $^{\mathrm{b}}$ & 1.00 & $1.29(0.97-1.71)$ & $1.53(1.15-2.03)$ & $1.93(1.43-2.61)$ \\
\hline
\end{tabular}

OR, odds ratio; CI, confidence interval; TyG, triglyceride glucose; HOMA-IR, homeostasis model assessment of insulin resistance.

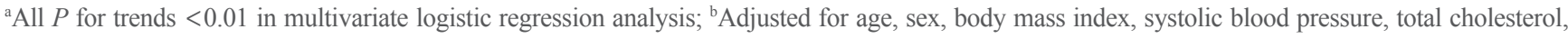
high density lipoprotein cholesterol, alanine aminotransferase, presence of hypertension.

IR at $0.672(95 \% \mathrm{CI}, 0.650$ to 0.694$)(P<0.01)$. These results suggest that regarding the prediction of NAFLD, the TyG index is superior to the HOMA-IR.

\section{DISCUSSION}

In the present study, following adjustment for conventional risk factors, an independent relationship between the TyG index and
NAFLD was observed. Furthermore, results demonstrated that the TyG index was superior in its ability to identify NAFLD compared with HOMA-IR. To the best of our knowledge, this is the first study wherein the diagnostic effectiveness of the TyG index and HOMA-IR for identifying NAFLD has been compared.

NAFLD is related to IR and metabolic syndromes associated with hyperinsulinemia, hypertriglyceridemia, and hyperglyce- 
mia [5,26-28]. Recently, Zheng et al. [29], even demonstrated that TyG index could predict an incidence of NAFLD in longitudinal, prospective cohort study. In agreement with previous studies, most of the metabolic risk factors evaluated by us increased or decreased according to the TyG index and the presence of NAFLD. In addition, unlike previous studies, which reported an association between the presence of NAFLD and the TyG index [22-24], we further examined the relationship between the severity of NAFLD and the TyG index. Results demonstrated that the severity of NAFLD was strongly associated with the TyG index.

IR has been shown to have an important pathological association with NAFLD. Furthermore, both the TyG index and HOMA-IR are well-known representative markers of IR. In the present study, Pearson's correlation analysis was performed and confirmed that there was a significant relationship between the TyG index and HOMA-IR $(r=0.466, P<0.001)$ (data not shown). Interestingly, we observed that the TyG index displayed a higher OR and AUC of the ROC curve than the HOMA-IR for predicting NAFLD. These results suggest that the TyG index is a superior surrogate marker for predicting NAFLD. Recent studies have also reported the superiority of the TyG index for identifying IR [21,30,31]. For example, Vasques et al. [21] reported that the TyG index was superior to HOMA-IR in identifying IR in a Brazilian population. Although the reason for this finding remains unknown, the underlying mechanism for the superiority of the TyG index over HOMA-IR in predicting NAFLD can be explained by the crucial roles of glucotoxicity and lipotoxicity play in the modulation of IR, which shows key pathological association with NAFLD [32-34]. Our findings are in line with those of recent studies that demonstrate the superiority of the TyG index to HOMA-IR for evaluating metabolic risk factors associated with IR (e.g., diabetes and subclinical atherosclerosis) [35-37]. In contrast, other studies have reported that the HOMA-IR primarily reflects IR in the liver $[38,39]$. The mechanisms associated with this relationship should be clarified in prospective large-scale studies.

The present study had the following limitations. (1) Since this was a cross-sectional observational study, a causality cannot be presumed from the results. (2) The participants comprised Korean adults from a single institution and most of subjects were non-obese, healthy population. For these reasons, levels of fasting insulin and HOMA-IR were relatively low compared with other previous studies. Thus, the generalizability of the results may be limited. (3) The TyG index was compared with the HOMA-IR rather than with the hyperinsulinemic-euglycemic clamp test as the gold standard for assessing IR. However, the IR index derived from a euglycemic clamp has been shown to correlate with the TyG index and HOMA-IR. (4) A liver biopsy with a histological examination was not performed, which is the gold standard technique for identifying steatosis. Moreover, ultrasound was used for assessing the presence of NAFLD in this study; however, ultrasonography, a first-line imaging technique, is a highly useful noninvasive technique that is often used in both clinical practice and epidemiological studies [40].

The results of the present study indicate that there is a significant association between the TyG index and the prevalence of NAFLD in Korean subjects. Moreover, the TyG index was superior to HOMA-IR in predicting NAFLD. The TyG index is a simple and cost-effective marker of IR and appears to be a useful marker for predicting NAFLD.

\section{CONFLICTS OF INTEREST}

No potential conflict of interest relevant to this article was reported.

\section{ACKNOWLEDGMENTS}

We would like to thank the Gangnam Severance Health Promotion Research team for supporting the construction of the registry of data from the Health Promotion Center of the Gangnam Severance Hospital.

\section{AUTHOR CONTRIBUTIONS}

Conception or design: J.S.P., S.B.L., M.K.K. Acquisition, analysis, or interpretation of data: S.K., K.P., J.H.K., S.J.B., J.S.N., C.W.A. Drafting the work or revising: S.B.L., M.K.K., J.S.P. Final approval of the manuscript: J.S.P.

\section{ORCID}

Sang Bae Lee https://orcid.org/0000-0002-2998-6534

Min Kyung Kim https://orcid.org/0000-0003-0150-3550

Jong Suk Park https://orcid.org/0000-0002-5385-1373

\section{REFERENCES}

1. Browning JD, Szczepaniak LS, Dobbins R, Nuremberg P, Horton JD, Cohen JC, et al. Prevalence of hepatic steatosis in an urban population in the United States: impact of eth- 
nicity. Hepatology 2004;40:1387-95.

2. Smits MM, Ioannou GN, Boyko EJ, Utzschneider KM. Nonalcoholic fatty liver disease as an independent manifestation of the metabolic syndrome: results of a US national survey in three ethnic groups. J Gastroenterol Hepatol 2013;28:664-70.

3. Simeone JC, Bae JP, Hoogwerf BJ, Li Q, Haupt A, Ali AK, et al. Clinical course of nonalcoholic fatty liver disease: an assessment of severity, progression, and outcomes. Clin Epidemiol 2017;9:679-88.

4. Ahmed A, Wong RJ, Harrison SA. Nonalcoholic fatty liver disease review: diagnosis, treatment, and outcomes. Clin Gastroenterol Hepatol 2015;13:2062-70.

5. Yki-Jarvinen H. Non-alcoholic fatty liver disease as a cause and a consequence of metabolic syndrome. Lancet Diabetes Endocrinol 2014;2:901-10.

6. Anstee QM, Targher G, Day CP. Progression of NAFLD to diabetes mellitus, cardiovascular disease or cirrhosis. Nat Rev Gastroenterol Hepatol 2013;10:330-44.

7. Vanni E, Bugianesi E, Kotronen A, De Minicis S, YkiJarvinen H, Svegliati-Baroni G. From the metabolic syndrome to NAFLD or vice versa? Dig Liver Dis 2010;42:32030 .

8. van den Berg EH, Amini M, Schreuder TC, Dullaart RP, Faber KN, Alizadeh BZ, et al. Prevalence and determinants of non-alcoholic fatty liver disease in lifelines: a large Dutch population cohort. PLoS One 2017;12:e0171502.

9. Lee JI, Kim MC, Moon BS, Song YS, Han EN, Lee HS, et al. The relationship between 10 -year cardiovascular risk calculated using the pooled cohort equation and the severity of non-alcoholic fatty liver disease. Endocrinol Metab (Seoul) 2016;31:86-92.

10. Birkenfeld AL, Shulman GI. Nonalcoholic fatty liver disease, hepatic insulin resistance, and type 2 diabetes. Hepatology 2014;59:713-23.

11. Machado M, Cortez-Pinto H. Non-alcoholic fatty liver disease and insulin resistance. Eur J Gastroenterol Hepatol 2005; 17:823-6.

12. Lee JH, Rhee PL, Lee JK, Lee KT, Kim JJ, Koh KC, et al. Role of hyperinsulinemia and glucose intolerance in the pathogenesis of nonalcoholic fatty liver in patients with normal body weight. Korean J Intern Med 1998;13:12-4.

13. Matthews DR, Hosker JP, Rudenski AS, Naylor BA, Treacher DF, Turner RC. Homeostasis model assessment: insulin resistance and beta-cell function from fasting plasma glucose and insulin concentrations in man. Diabetologia 1985;28:412-9.
14. Brunt EM, Kleiner DE, Wilson LA, Belt P, NeuschwanderTetri BA; NASH Clinical Research Network (CRN). Nonalcoholic fatty liver disease (NAFLD) activity score and the histopathologic diagnosis in NAFLD: distinct clinicopathologic meanings. Hepatology 2011;53:810-20.

15. Pais R, Charlotte F, Fedchuk L, Bedossa P, Lebray P, Poynard $\mathrm{T}$, et al. A systematic review of follow-up biopsies reveals disease progression in patients with non-alcoholic fatty liver. J Hepatol 2013;59:550-6.

16. European Association for the Study of the Liver (EASL); European Association for the Study of Diabetes (EASD); European Association for the Study of Obesity (EASO). EASL-EASD-EASO clinical practice guidelines for the management of non-alcoholic fatty liver disease. J Hepatol 2016;64:1388-402.

17. Isokuortti E, Zhou Y, Peltonen M, Bugianesi E, Clement K, Bonnefont-Rousselot D, et al. Use of HOMA-IR to diagnose non-alcoholic fatty liver disease: a population-based and inter-laboratory study. Diabetologia 2017;60:1873-82.

18. Salgado AL, Carvalho Ld, Oliveira AC, Santos VN, Vieira JG, Parise ER. Insulin resistance index (HOMA-IR) in the differentiation of patients with non-alcoholic fatty liver disease and healthy individuals. Arq Gastroenterol 2010;47: 165-9.

19. Simental-Mendia LE, Rodriguez-Moran M, GuerreroRomero F. The product of fasting glucose and triglycerides as surrogate for identifying insulin resistance in apparently healthy subjects. Metab Syndr Relat Disord 2008;6:299-304.

20. Guerrero-Romero F, Simental-Mendia LE, Gonzalez-Ortiz M, Martinez-Abundis E, Ramos-Zavala MG, HernandezGonzalez SO, et al. The product of triglycerides and glucose, a simple measure of insulin sensitivity. Comparison with the euglycemic-hyperinsulinemic clamp. J Clin Endocrinol Metab 2010;95:3347-51.

21. Vasques AC, Novaes FS, de Oliveira Mda S, Souza JR, Yamanaka A, Pareja JC, et al. TyG index performs better than HOMA in a Brazilian population: a hyperglycemic clamp validated study. Diabetes Res Clin Pract 2011;93:e98-100.

22. Simental-Mendia LE, Simental-Mendia E, Rodriguez-Hernandez H, Rodriguez-Moran M, Guerrero-Romero F. The product of triglycerides and glucose as biomarker for screening simple steatosis and NASH in asymptomatic women. Ann Hepatol 2016;15:715-20.

23. Zhang S, Du T, Zhang J, Lu H, Lin X, Xie J, et al. The triglyceride and glucose index (TyG) is an effective biomarker to identify nonalcoholic fatty liver disease. Lipids Health 
Dis $2017 ; 16: 15$.

24. Zhang S, Du T, Li M, Jia J, Lu H, Lin X, et al. Triglyceride glucose-body mass index is effective in identifying nonalcoholic fatty liver disease in nonobese subjects. Medicine (Baltimore) 2017;96:e7041.

25. Saadeh S, Younossi ZM, Remer EM, Gramlich T, Ong JP, Hurley $\mathrm{M}$, et al. The utility of radiological imaging in nonalcoholic fatty liver disease. Gastroenterology 2002;123:74550.

26. Byrne CD, Targher G. NAFLD: a multisystem disease. J Hepatol 2015;62:S47-64.

27. Abate N, Chandalia M. Risk of obesity-related cardiometabolic complications in special populations: a crisis in Asians. Gastroenterology 2017;152:1647-55.

28. Kim D, Kim WR. Nonobese fatty liver disease. Clin Gastroenterol Hepatol 2017;15:474-85.

29. Zheng R, Du Z, Wang M, Mao Y, Mao W. A longitudinal epidemiological study on the triglyceride and glucose index and the incident nonalcoholic fatty liver disease. Lipids Health Dis 2018;17:262.

30. Guerrero-Romero F, Villalobos-Molina R, Jimenez-Flores JR, Simental-Mendia LE, Mendez-Cruz R, Murguia-Romero $\mathrm{M}$, et al. Fasting triglycerides and glucose index as a diagnostic test for insulin resistance in young adults. Arch Med Res 2016;47:382-7.

31. Du T, Yuan G, Zhang M, Zhou X, Sun X, Yu X. Clinical usefulness of lipid ratios, visceral adiposity indicators, and the triglycerides and glucose index as risk markers of insulin resistance. Cardiovasc Diabetol 2014;13:146.

32. Taniguchi A, Fukushima M, Sakai M, Kataoka K, Nagata I, Doi K, et al. The role of the body mass index and triglyceride levels in identifying insulin-sensitive and insulin-resistant variants in Japanese non-insulin-dependent diabetic patients. Metabolism 2000;49:1001-5.

33. Taniguchi A, Nakai Y, Sakai M, Yoshii S, Hamanaka D, Ha- tae Y, et al. Relationship of regional adiposity to insulin resistance and serum triglyceride levels in nonobese Japanese type 2 diabetic patients. Metabolism 2002;51:544-8.

34. Bergmann K, Sypniewska G. Diabetes as a complication of adipose tissue dysfunction. Is there a role for potential new biomarkers? Clin Chem Lab Med 2013;51:177-85.

35. Lee SH, Kwon HS, Park YM, Ha HS, Jeong SH, Yang HK, et al. Predicting the development of diabetes using the product of triglycerides and glucose: the Chungju Metabolic Disease Cohort (CMC) study. PLoS One 2014;9:e90430.

36. Irace C, Carallo C, Scavelli FB, De Franceschi MS, Esposito $\mathrm{T}$, Tripolino $\mathrm{C}$, et al. Markers of insulin resistance and carotid atherosclerosis: a comparison of the homeostasis model assessment and triglyceride glucose index. Int J Clin Pract 2013;67:665-72.

37. Kim MK, Ahn CW, Kang S, Nam JS, Kim KR, Park JS. Relationship between the triglyceride glucose index and coronary artery calcification in Korean adults. Cardiovasc Diabetol 2017;16:108.

38. Tripathy D, Almgren P, Tuomi T, Groop L. Contribution of insulin-stimulated glucose uptake and basal hepatic insulin sensitivity to surrogate measures of insulin sensitivity. Diabetes Care 2004;27:2204-10.

39. Bonora E, Targher G, Alberiche M, Bonadonna RC, Saggiani $\mathrm{F}$, Zenere MB, et al. Homeostasis model assessment closely mirrors the glucose clamp technique in the assessment of insulin sensitivity: studies in subjects with various degrees of glucose tolerance and insulin sensitivity. Diabetes Care 2000;23:57-63.

40. Loria P, Adinolfi LE, Bellentani S, Bugianesi E, Grieco A, Fargion S, et al. Practice guidelines for the diagnosis and management of nonalcoholic fatty liver disease. A decalogue from the Italian Association for the Study of the Liver (AISF) Expert Committee. Dig Liver Dis 2010;42:272-82. 